
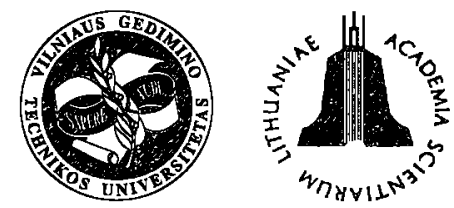

ISSN 1648-4142 TRANSPORT

http:/www.vtu.It/english/editions

TRANSPORT - 2003, Vol XVIII, No 4, 157-161

\title{
MODELLING RELATIONS BETWEEN TRANSPORTATION OBJECTS USING DISTRIBUTED ATTRIBUTE SETS
}

\author{
Rolandas Griškevičius \\ Dept of Fundamental Sciences, Vilnius Gediminas Technical University, \\ Sauletekio al.11,LT-2040 Vilnius, Lithuania.E-mail: rolandas.griskevicius@fm.vtu.lt
}

Received 200301 16; accepted 20030630

\begin{abstract}
Relational sets with distributed attributes (RSDA), described in this paper, are used to model relationships between transportation objects. RSDA is a data storage and management model, extending traditional model of E. F. Codd. It has a richer attribute semantics and intersection effect. Because relationships between transportation objects have rich semantics (i.e. relationship between a cargo and a means of transport), special methodology is required to capture relation singularities.
\end{abstract}

Keywords: relational sets, business object, object, semantic relationships.

\section{Introduction}

One of the ways to extend attribute semantics in a traditional Codd's [1] model is to join several sets. For example, two relational sets $(R S)$ are given: $R 1\left(A, B_{11}\right.$ $\left.\ldots B_{1 \mathrm{n1}}\right)$ and $R 2\left(A, B_{21} \ldots B_{2 \mathrm{n} 2}\right)$, with dependency $A \rightarrow$ $\left\{B_{11}, \ldots B_{1 \mathrm{n} 1}\right\}, A \rightarrow\left\{B_{21} \ldots B_{2 \mathrm{n} 2}\right\}$. Joined set, combined from both $R 1$ and $R 2$ attributes in its tuples, and only those tuples, where attributes $A$ from $R 1$ and $R 2$ are equal is as follows: $R 1>_{(A 1=A 2)} R 2=R 3\left(A, B_{11}, \ldots B_{1 n 1}, B_{21} \ldots\right.$ $\left.B_{2 n 2}\right)$. From the functional dependency view $([2-5])$, join operation extends attribute $A$ semantics, because in $R 3$ attributes A functional dependency becomes $A \rightarrow\left\{B_{11}\right.$, $\left.\ldots B_{1 n 1}, B_{21} \ldots B_{2 n 2}\right\}$. In this case, attributes $B_{11}, \ldots$ $B_{1 n 1}, \mathrm{~B}_{21} \ldots \mathrm{B}_{2 n 2}$ can be considered as semantic components of attribute $A$. By applying relational operator "left outer join", additional semantics can be extracted: this operator yields an empty part of a tuple, when corresponding attribute in a joined set does not exist. In general the resulting set is as follows: $R_{m}=\left(A, B_{i 1} \ldots B_{m n}\right)$. Such a set will have dependent attributes as domains and tuple keys as corteges (Fig 1).

From a semantic point of view each value of attribute $B_{1} \ldots B_{n}$ functionally depends on tuple key a, thus forming complex value from subattributes $<a>$ and $\langle B\rangle$. This adds new functionality to the set: 1) Each

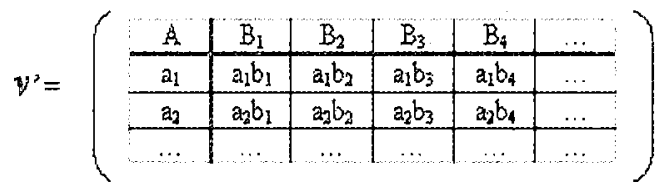

Fig 1. Prototype of relational set with distributes attributes value may have its own semantics, 2) attribute values are distributed, but undistinguishable in mean of semantics, 3) for each attribute an empty value $\varnothing$ may exist, which shows that the given attribute has no meaning in an applied domain.

The approach of an attribute distribution was proposed by $P$. Adomenas ([6, 7]). We will call these sets as "relational sets with distributed attributes" (RSDA).

From the view of data, there's no difference between subattributes $\langle a\rangle$ and $\langle B\rangle$, so in a general case RSDA set may be denoted as (Fig 2):

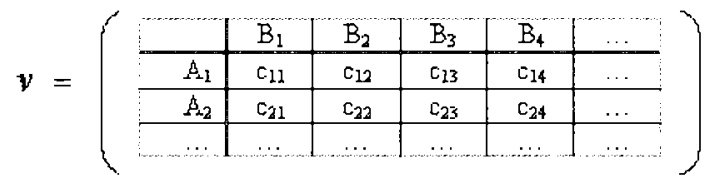

Fig 2. Relational set with distributed attributes

Subattribute sets $\langle A\rangle=A_{1} \ldots A_{k}$ and $\langle B\rangle=B_{1}$.. $B_{l}$ form subshemas, which later form the schema of a set $v: R_{v}=V(\langle A\rangle ;\langle B\rangle)$. Numbers $k=|\langle A\rangle|, l=|\langle B\rangle|$ show the count of subattributes in the subschemas. Under RSDA attribute we will mean any pair of subattributes $A B_{j}$. Under attribute value we will mean certain evaluation of attribute (i.e. number, character string), expressed in the form acceptable by calculation. Thus, value and subattribute pair fully describe an attribute. If $i=1 \ldots$ $|<A>|$ ir $j=1 \ldots \mid\langle B>|$, then $\left\langle c_{i j}\right\rangle=\operatorname{attr}(V)$ - attribute entirety of a given set. Map $s u b:\left\langle c_{i j}\right\rangle \rightarrow\langle A\rangle$ shows dependency of attribute to a corresponding subattributes. In a general case, RSDA is a triple: $v=\langle V,\langle c\rangle, \operatorname{sub}(A)\rangle$, 
here $V$-schema, , $\langle c\rangle$ - atribute entirety, sub - attribute dependency map. It is possible to have $|\angle A>|=0$ or $|<B>|=0$, then $\operatorname{attr}(V)=\varnothing$.

For some cases, the series $v_{l} \ldots v_{n}$ with the same subschemas: $\langle A\rangle_{1}=\langle A\rangle_{2} \ldots=\langle A\rangle_{n}$ and $\langle B\rangle_{1}=$ $\langle B\rangle_{2} \ldots=\langle B\rangle_{n}$ are used. Furthermore, given series address the same object and only different its data. Then $v^{[n]}=v_{1} \downarrow_{v_{2}} \downarrow \ldots \downarrow v_{n}$ is the definition of a multiattribute set. Here $\downarrow^{2}$ - folding operator. Similarly $v^{[n] \uparrow i}=v_{i}, 1<i$ $<n$, here $\Uparrow^{i}-$ unfolding operator. An attribute of the set $\mathrm{v}^{[\mathrm{n}]}$ contains the series $\left\langle c^{1} \ldots c^{n}>\right.$. Folding and unfolding operators do not change the schema of the set, though they change multiattribute series $\left\langle c^{1} \ldots c^{n}\right\rangle$.

Attribute access operator $v^{[n] \uparrow i}=v_{i}$ enables to access particular attribute in the multiattribute set. It doesn't change either subschema or the count of multiattribute series $n$.

\section{Operations in the Distributed Attribute Envi- ronment}

\subsection{Primitive change operator}

RSDA model contains only 1 primitive operator, which has the following form: $C H(S U B 1, S U B 2, V A L U E)$. Here: $S U B 1$ and $S U B 2$ - subattributes. VALUE - denotes value of an attribute. $C H$ can operate in 3 modes: change, insert and delete. In the first case operator $\mathrm{CH}$ changes the value of an attribute, referred by subattributes $S U B I$ and $S U B 2$ (attribute $S U B 1 / S U B 2$ ). If there were no attribute before, $C H$ inserts either one or both subattributes and value for a newly created attribute. (insert mode). All new attributes which have the newly created subattribute in their pairs are empty, except one - referred by $S U B 1 / S U B 2$. When $C H$ with $V A L U E=\varnothing$ is invoked, the value of an attribute is deleted (delete mode). If subattribute contains all $\varnothing$ after the deletion of the attribute, it is also deleted.

\subsection{Relational RSDA operators}

As in a traditional relation model, similar relation operators exist for RSDA:

Projection: $\pi_{(\langle A\rangle,\langle B\rangle)}$ - produces a set from some other set by pulling out only the identified subattributes in subschemas.

Selection: $\sigma_{(\{\forall \mid \exists \exists c \theta a)}$ - produces a set containing only those attributes, which match specified criteria. $\theta$ any of comparison operators, for example: $<,>, \leq, \geq$, $\neq,=$.

Cartesian: produces a set from 2 sets, containing all possible attribute combinations from each set: $v^{[2]}=v_{1} \times$ $v_{2 .}$

Intersection operator is expressed as: $v_{1} \triangleright \triangleleft_{\{A 1 \theta A 2\}}$ $v_{2}$, here $\mathrm{A} 1$ and $\mathrm{A} 2$ are subattributes of the first and second RSDA operands, $\theta$ - any comparison operator (i.e.
$<,>, \leq, \geq, \neq,=$ ). This operator is not a primitive, it can be expressed using selection, projection and Cartesian product.

Union operator yields a set, which is combined from two sets by "sticking" them together on one specified subschema. It is of the following form: $v_{1} \cup_{\{S 1, S 2\}} v_{2}$, here S1 and S2 denote subschemas on which to apply the union operation.

Subschema transposition operator reverses the order of subschemas: $\nabla V(\langle A\rangle ;\langle B\rangle)=V(\langle B\rangle ;\langle A\rangle)$.

\subsection{RSDA scalar operations}

Scalar operation for the given RSDA series $\langle V\rangle=$ $v_{1} \ldots v_{n}$ with the schemas $V(\langle A\rangle,\langle B\rangle)$ is as follows: $\mathrm{c}_{i j}{ }^{\mathrm{r}}=\operatorname{scal}\left(c_{i j}{ }^{1}, \ldots c_{i j}{ }^{k}\right), i=1 \ldots\left|<A>^{*}\right|, j=1 \ldots$ $\mid<B>$, here scal (..) - prefix form of any scalar relation, $\left.\angle A\rangle^{*}=\angle A\right\rangle^{1}\left\lceil\ldots \ldots \mid\langle A\rangle^{k},\langle B\rangle^{*}=\langle B\rangle^{1}\lceil\right.$ $\ldots \prod<\|^{k},<c_{i j}>=\operatorname{attr}\left(V\left(<A>^{*},<B>^{*}\right)\right.$.

\subsection{Transformations RS $\rightarrow$ RSDA and RS' $\rightarrow$ RSDA}

Transformation RS $\rightarrow$ RSDA is possible when a system of related RS is in the $3^{\text {rd }}$ normal form and there is common key attribute(s) $A$ in every RS. By applying grouping operator $\gamma[8]$, we find all existing domain values of $A$. This grouped set later is joined with sets $r_{1} \ldots r_{n}$ using LEFT OUTER JOIN operator. Transformation expression is as follows:

$$
\begin{aligned}
& t_{R S \rightarrow R S D A}={ }_{A} \gamma\left(\pi_{A}\left(r_{1}\right) \cup \ldots\right. \\
& \left.\cup \pi_{A}\left(r_{n}\right)\right) \\
& >_{A} r_{1}>_{A} \ldots>_{A} r_{n} .
\end{aligned}
$$

The reverse transformation, based on division ( $[9$, 10]), is more complex, as after certain processing some attributes of RS s may not exist. This is a special case and is not covered in this paper.

To simulate RSDA using a traditional relational set system, or to save RSDA to RS we need to express the first in terms of traditional relational algebra. The exported set will have a special schema, functionally very close to RSDA. We consider it as RSDA-ready set, or RS. Such a set, data of which is taken from Fig 2 is shown in Fig 3:

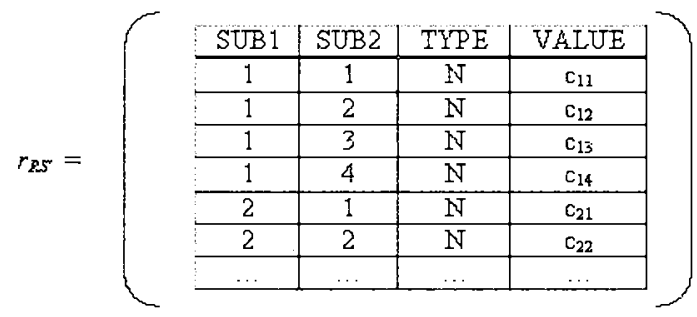

Fig 3. RSDA-ready set 
Attributes SUB1 and SUB2 contain the references of RSDA subattributes. Attribute VALUE contains values of certain attributes. Attribute TYPE contains a type code attribute which depends on implementation.

Transformation then from RS' to RSDA is as follows:

$$
\begin{aligned}
& t_{R A^{\prime} \rightarrow R P A}=_{S U B 1} \gamma\left(\pi_{S U B 1}\left(r_{R A^{\prime}}\right)\right)> \\
& S_{S U B 1}\left(\pi_{S U B 1, V A L U E} \sigma_{S U B 2=\mathrm{H}_{1}}\left(r_{R A^{\prime}}\right) \ldots\right. \\
& >_{S U B 1}\left(\pi_{S U B 1, V A L U E} \sigma_{S U B 2=\mathrm{H}_{p}}\left(r_{R A^{\prime}}\right)\right)
\end{aligned}
$$

here $H_{1} \ldots H_{p}={ }_{S U B 2} \gamma\left(\pi_{S U B 2}\left(r_{R A}\right)\right)$ - the set of SUB2 values with duplicates eliminated.

The aim of this transformation is to form the traditional algebra set, structurally similar to RSDA. Transformation is based on SUB1 and SUB2 attributes.

Using this way of transformation, desired sequence of traditional relational algebra operations can be applied on RS', and later RS' can be presented in look-like RSDA view. We call it RS' - based RSDA calculus. Primitive operator $\mathrm{CH}$, described previously, can easily be expressed using primitive relational algebra operators $\mathrm{CH}$, $D E L$ and $A D D$ for RS'.

\subsection{Integration of RSDA and traditional rela- tional sets}

The RSDA-like expression form Fig 3 and the transformation 1 give possibility to join traditional and RSDA sets into one relational set system. Two types of joins can exist, in distinction where data is carried: subattribute renaming join (S-join) and attribute append join (A-join).

\begin{tabular}{|c|c|c|c|c|c|c|c|c|c|c|}
\hline$\ldots$ & Sumber & ID & \multirow{3}{*}{$S$} & & $A !$ & $\mathrm{A} 2$ & \multirow{4}{*}{$=$} & \multirow[b]{2}{*}{$\mathrm{ABC} 001$} & $A !$ & $\mathrm{A2}$ \\
\hline ... & $A B C O 01$ & $\mathrm{B!}$ & & $B !$ & $c_{11}$ & 912 & & & द1! & 9.12 \\
\hline$\ldots$ & $A B A O D 2$ & $\bar{B} 2$ & & $B 2$ & $c_{21}$ & 0 & & AAAO02 & 021 & 223 \\
\hline$\ldots$ & BBB 111 & B3 & & $\overline{B 3}$ & $c_{31}$ & $c_{32}$ & & BDE III & 31 & 832 \\
\hline
\end{tabular}

The S-join $v_{S}=r_{1}>{ }_{\{R, K, S m\}} v_{2}$ and A-join $v^{[2]}{ }_{S}=$ $r_{1}>_{\{R, K, S m\}}^{A} \nu_{2}$ operators (Fig 4, Fig 5):

\begin{tabular}{|c|c|c|c|c|c|c|c|c|c|c|}
\hline ... & Number & ID & & & $\mathrm{Al}$ & $\mathrm{A2}$ & & & A! & A2 \\
\hline ... & $\mathrm{ABCOO}$ & $\mathrm{B1}$ & $A$ & B! & $c_{11}$ & 612 & & 31 & 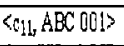 & $\varepsilon_{013}$ \\
\hline ... & AAACO2 & 82 & $\Omega$ & $\mathrm{B} 2$ & 621 & $\mathrm{~cm}_{23}$ & $=$ & 32 & $\langle 21$, SAA OOA2〉 & $\varsigma_{23}$ \\
\hline ... & BEB III & $\mathrm{B} 3$ & & B3 & 331 & 652 & & 83 & $\left\langle{ }_{23,}, \mathrm{BEB} \| 11\right\rangle$ & $8 c_{32}$ \\
\hline
\end{tabular}

Fig 4. S-join example

Fig 5. A-join example

3. Use of RSDA to Model Relationships between Transportation Objects

3.1. Modelling of relationship data of transportation objects

As RSDA's attribute is the intersection of subattributes, it can be used to model the intersections of ob- jects. Attribute at the intersection holds some value, thus defining data of the intersection. This intersection with value defines relationship.

Schedule is the kind of relation between some objects. As objects to relate we will choose vehicles from set $\operatorname{Veh}(V I D, N o, W g h t)$ with identifier, number and maximal transportation weight and cargos from set Cg(CID, Name, Wght ) with identifier, name, weight. Schedule will be presented as RSDA $v^{[2]}$, here $v^{[2]} \uparrow^{1}-$ cargo load time and $v^{\left(2 / \uparrow^{2}\right.}$-cargo unload time. Schema of latter: $S c h^{[2]}(\langle V I D\rangle,\langle C I D\rangle)$, here subschema $\langle V I D>$ will correspond vehicle identifiers, subschema $\angle C I D>$ - cargo identifiers.

Using operator $\mathrm{CH}$ schedule time can be entered. Then the following data checks may be applied:

Scalar operation $v=S c h^{/ 2 J \uparrow} \uparrow^{l}<S c h^{2 / \uparrow^{2}}$ yields RSDA with binary attributes, false value of which denotes wrong time entered, as unload time cannot be less than load time.

Selection $v=\sigma_{(\forall c=F A L S E)} v$ produce the set, containing these wrong attributes.

Cargo weight control. Cargo weight cannot exceed it's vehicle maximum transportation weight.

First, temporary set with cargo and vehicle weight, produced:

$\quad \mathcal{v}^{[2]}=V e h>^{A}{ }_{\{W g h t, V I D,<V I D>\}}\left(C g>^{A}{ }_{W g h t, C I D,}<C I D>\right.$;
$\left.S C h^{[2] \uparrow 1}\right)$.

Then scalar operation $v=v^{[2]^{\uparrow 1}}<v^{[2] \uparrow 2}$ gives the set with inadequate cargos and vehicles.

Time intersection control is the most important schedule operation. One or more schedules can be generated, but we always want to ensure that their time does not intersect.

Using Cartesian product we first generate temporary set:

$v^{[4]}=\operatorname{Sch} 1^{[2]} \times \operatorname{Sch} 2^{[2]}$

It can be now checked for time intersection using scalar operation:

$\nu=$ date_test $\left(S \operatorname{Sch} 1^{[2] \uparrow 1}, \operatorname{Sch} I^{[2] \uparrow 1}, \operatorname{Sch} 1^{[2] \uparrow 1}\right.$, $\left.\operatorname{Sch} 1^{[2]^{\uparrow 2}}\right)$, here

date_test $\left(v_{1}, v_{2}, v_{3}, v_{4}\right)=\operatorname{between}\left(v_{3}, v_{1}, v_{2}\right)$ and between $\left(v_{4}, v_{1}, v_{2}\right)$,

between $\left(v_{3}, v_{1}, v_{2}\right)=v 1 \leq v 3 \leq v 2$, here $\leq$ - scalar comparison.

Visualisation of schedule. The S-join generates visual representation of schedule: $S c h$
$\operatorname{Sch}^{[2] \uparrow 1}$ ).

$\operatorname{Sch}^{[2] \uparrow 1}=V e h>S_{\{N o, V I D,<V I D>\}}\left(C g>S_{\{N a m e, C I D,<C I D>\}}\right.$

\subsection{Modelling relationship semantics of transporta- tion objects}

Sets of vehicles and cargos and RSDA set of relationships between them are given in Fig 6. The latter is expressed in RSDA-ready form using standard means of 
relational sets. Subattributes sub1 and sub2 relate to cargo identifiers CID and vehicle identifiers VID correspondingly.

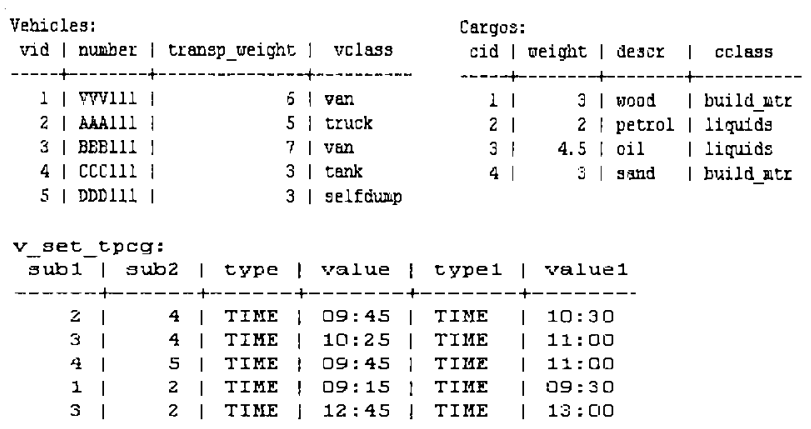

Fig 6. Relational set with distributed attributes "v_set_tpcg"

Definitions of semantic rules between transportation objects Vehicles and Cargos are in the conjugated form of Horn clauses:

$\left(\underline{A}_{1} \rightarrow B_{1}\right) \& \ldots \&\left(\underline{A}_{n} \rightarrow B_{n}\right) \rightarrow(\underline{C} \rightarrow D)$,

here $C=C_{1} \& \ldots \& C_{l}$, operations $\rightarrow$ and \& - denote implication and conjunction. Upper definition shows the way of getting result $\mathrm{D}$ from clause set $\mathrm{C}$ and rules sets $A_{l} \rightarrow B_{1} \ldots A_{n} \rightarrow B_{n}$. Table shows Horn clauses expressed in terms of Prolog language:

Capturing semantics between Vehicle and Cargo objects

\begin{tabular}{|c|c|}
\hline Prolog clauses & Comments \\
\hline $\begin{array}{l}\text { attribute_correct(Cid, Vid } \\
\text { ) :- } \\
\text { get_data_by_sub( Cid, Vid, } \\
\text { Cclass, Vclass, Cweight, } \\
\text { Vweight), } \\
\text { vehicles( Vid, _, Vweight, } \\
\text { Vclass ), } \\
\text { cargos ( Cid, Cweight, -' } \\
\text { Cclass), } \\
\text { weight_correct ( Vweight, } \\
\text { Cweight), } \\
\text { vehicle_correct( Vclass, } \\
\text { Cclass). }\end{array}$ & $\begin{array}{l}\text { Weight and class } \\
\text { extraction using } \\
\text { predicates } \\
\text { "get_data_by_su } \\
\text { b" (extracts data } \\
\text { from RSDA set), } \\
\text { "vehicles", } \\
\text { "cargos". }\end{array}$ \\
\hline $\begin{array}{l}\text { weight_correct( } \mathrm{Vw}, \mathrm{Cw} \text { ) } \\
:- \\
\mathrm{Vw}<\mathrm{Cw}, \\
\text { write('Weight } \\
\text { incorrect:'), } \\
\text { write(Vw), write('<'), } \\
\text { write(Cw), nl, fail. } \\
\text { weight_correct( } \mathrm{Vw}, \mathrm{Cw} \text { ) } \\
:- \\
\mathrm{Vw}_{\mathrm{w}}>=\mathrm{Cw} .\end{array}$ & $\begin{array}{l}\text { Predicate to } \\
\text { control weight } \\
\text { of cargo and } \\
\text { allowable } \\
\text { transportation } \\
\text { weight of } \\
\text { vehicle. If latter } \\
\text { is less, then } \\
\text { predicate fails } \\
\text { with an error. }\end{array}$ \\
\hline
\end{tabular}

\begin{tabular}{|c|c|}
\hline $\begin{array}{l}\text { class_correct ( VV,CC). } \\
\text { class_correct ( } \\
\text { liquids }) . \\
\text { class_correct ( } \\
\text { build_mtr ). } \\
\text { class_correct ( } \\
\text { build_mtr ). }\end{array}$ & $\begin{array}{l}\text { Definitions of } \\
\text { cargo and } \\
\text { vehicle classes. } \\
\text { Classes are used } \\
\text { to control } \\
\text { suitable cargo } \\
\text { transportation. } \\
\text { For example, } \\
\text { only tank can } \\
\text { transport liquids. } \\
\text { In this form we } \\
\text { define required } \\
\text { semantics of } \\
\text { relation. }\end{array}$ \\
\hline $\begin{array}{l}\text { vehicle_correct( } \mathrm{VV}, \mathrm{CC}) \\
:- \\
\text { I+ class_correct( VV,CC), } \\
\text { write('Class is incorrect: } \\
\text { '), } \\
\text { write(VV),write('- } \\
\text { '),write(CC),fail. } \\
\text { vehicle_correct( VV, CC ' } \\
\text { :- }\end{array}$ & $\begin{array}{l}\text { Class-based } \\
\text { control of } \\
\text { vehicle - cargo } \\
\text { suitability. If } \\
\text { vehicle class } \\
\text { does not fall } \\
\text { under cargo's } \\
\text { class, predicate } \\
\text { fails with an } \\
\text { error. }\end{array}$ \\
\hline
\end{tabular}

Following Prolog query (Fig 7) tests relation validity. Test fails as by definition a truck cannot carry liquids.
I ?- attribute_correct $(3,2)$.
Class is incorrect: truck-liquids
no

Fig 7 Prolog test of relation validity

\section{Conclusions}

RSDA automatically guarantees object relationship uniquiness (in chapter 3.1 example relationship between cargo and vehicle is unique), as no same vehicles with the same cargo can appear as different subattributes. But for the same objects several RSDA sets can appear (several schedules in chapter 3.1 example). Then the optimal one can be chosen comparing them using RSDA algebra.

Rich semantics of relationships between transportation objects is captured using rule-based framework. In conjunction with relational RSDA algebra we get a full mechanism of relationships management (data and semantics can be managed). As RSDA sets can be integrated with traditional relational set management systems (using A-join and S-join operations), we get also open architecture of framework, which can be implemented on any transportation management system based on relational set management system. 


\section{References}

1. Codd, E. F. A Relational Model Of Data For Large Shared Data Banks, CACM 1970 - 13(6).

2. Armstrong, W. W. Dependency structure of database relationships. In: Proceedings IFIP, North Holland, Amsterdam, 1974 , p. $580-583$

3. Binemann-Zdanowicz, A. Systematization of Approaches to Equality-Generating Constraints. ADBIS-DASFAA 2000, p. 307-314.

4. Doležal, T. Cardinality constraints for n-ary relationship types. In ADBIS'99, Maribor' 99.

5. McAllister, A. Complete rules for n-ary relationship cardinality constraints. Data and knowledge Engineering 27, 1998 , p. $255-288$.
6. Adomènas, P. Data functional feature sets and their adaptability. In: ADBIS'2001' . Research communications. ISBN 9986-05-449-4. Vilnius: Technika, 2001, p. 131-140.

7. Adomenas, P.; Ciucelis, A. Data Aggregation Sets in Adaptive Data Model. Informatica, 2002, Vol 13, No 4, p. 381 392.

8. Klug, A. Equivalence of relational algebra and relational calculus query languages having aggregate functions. Journal of the $A C M, 29(3), 1982$, p. 699-717.

9. Graefe, Goetz; Cole, Richard L. Fast Algorithms for Universal Quantification in Large Databases. TODS, 20(2), 1995, p. 187-236.

10. Desharnais, Jules; Jaoua, Ali; Mili, Fatma; Boudriga, Noureddine; Mili, Ali. A Relation Division Operator: The Conjugate Kernel., TCS 114(2), 1993, p. 247-272. 